\title{
Genetic and Environmental Influences on Serum Lipid Tracking: A Population-Based, Longitudinal Chinese Twin Study
}

\author{
SHANCHUN ZHANG, XIN LIU, JONATHAN NECHELES, HUI-JU TSAI, GUOYING WANG, BINYAN WANG, HOUXUN XING, \\ ZHIPING LI, XUE LIU, TONGHUA ZANG, XIPING XU, AND XIAOBIN WANG
}

\begin{abstract}
Department of Pediatrics [S.Z., X.L., J.N., H.-J.T., G.W., B.W., X.W.], Children's Memorial Hospital, Children's Memorial Research Center, Feinberg School of Medicine, Northwestern University, Chicago, Illinois 60614; Institute for Biomedicine [H.X., Z.L., X.L., T.Z.], Anhui Medical University, Hefei, Anhui 230032, China; Center for Population Genetics [X.X.], University of Illinois at Chicago School of Public Health, Chicago, Illinois 60612; Department of Epidemiology and Health Statistics [S.Z.], School of Medicine, Zhejiang University, Hangzhou, Zhejiang 310058, China
\end{abstract}

\begin{abstract}
We conducted cross-sectional and longitudinal twin analysis to explore genetic and environmental contribution to serum lipid tracking during childhood and adolescence. The study sample was part of a population-based twin cohort that was recruited in the rural areas of the Anhui Province of China. The baseline recruitment of twins was carried out from 1998 through 2000 and the follow-up from 2005 through 2007. Serum lipids showed significant tracking during childhood and adolescence. Participants with lipids at the highest tertile at the baseline tended to remain high at follow-up across ages and Tanner stages, whereas subjects with lipids at the lowest tertile at the baseline tended to remain low at follow-up. Using twin modeling, we showed that genetic and environmental factors contributed to individual variations in lipid levels and tracking from the baseline to the follow-up visit. The estimated tracking correlations for total cholesterol, triglyceride, and LDL cholesterol were in the range of $0.25-0.53$ and were predominantly influenced by genetic factors. In contrast, the phenotypic tracking of HDL cholesterol was influenced by both genetic and environmental factors. Our study underscores the importance of considering both environmental and genetic factors in studying the etiology of dyslipidemia. (Pediatr Res 68: 316-322, 2010)
\end{abstract}

$\mathrm{N}^{\mathrm{u}}$ umerous studies have demonstrated that serum lipid levels and lipoproteins influence the risk of cardiovascular diseases (1-3). Aortic and coronary fatty streaks, the early stages of atherosclerosis, have been observed in young (2-15 y) children $(3,4)$ and are associated with increased serum cholesterol levels (4). Significant tracking of serum lipids during childhood and from childhood to adulthood have been described $(5,6)$. However, there are limited data exploring the childhood to adolescence transition, which is accompanied by rapid body size growth, sexual maturation, and significant hormonal changes. These rapid changes likely contribute to individual variations in serum lipids and the subsequent risk of dyslipidemia in adulthood. A better understanding of the

Received May 19, 2009; accepted May 12, 2010.

Correspondence: Xiaobin Wang, M.D., Sc.D., Mary Ann and J. Milburn Smith Child Health Research Program, Department of Pediatrics, Children's Memorial Hospital, Children's Memorial Research Center, Feinberg School of Medicine, Northwestern University, Chicago, IL 60614; e-mail: xbwang@ childrensmemorial.org

Supported in part by a grant from the National Institute of Child Health and Human Development (R01 HD049059); the National Heart, Lung, and Blood Institute (R01 HL0864619), the National Institute of Aging (R01 AG032227), and the National Center for Research Resources (UL1RR025741). individual variations in serum lipids, its tracking, and the determinants from childhood to adolescence could inform future interventions that aim to prevent or mitigate dyslipidemia and cardiovascular diseases in later life.

There is growing evidence that serum lipid levels are influenced by both environmental factors such as diet (7) and physical activity $(7,8)$ and genetic factors $(9-12)$. Two longitudinal twin studies in Caucasians have provided evidence that there are age-specific genes controlling serum lipid variations during adolescence $(10,13)$. However, little is known about the genetic and environmental influences on serum lipid levels and the tracking from childhood to adolescence in Chinese, which constitutes one-fifth of the world population. Over the past decade, China has experienced rapid economic, nutritional, and lifestyle changes, which have been accompanied by rapidly rising rates of dyslipidemia and associated diseases such as obesity, atherosclerosis, type 2 diabetes, and the metabolic syndrome (14). Compared with western populations, the rural Chinese population provides a unique opportunity to gain insight into the interplay of genetic and environmental factors on serum lipid profiles and tracking patterns.

Using a unique twin design, the objectives of this study were to describe the degree of serum lipid tracking from childhood through adolescence and to estimate environmental and genetic contributions to the longitudinal lipid tracking in rural Chinese.

\section{MATERIALS AND METHODS}

Study sample. The study sample is part of a population-based twin cohort that was recruited in the Anqing and Luan areas of the Anhui Province of China. The baseline recruitment of twins was from September 1998 through May 2000, and the follow-up was from August 2005 through February 2007.

These areas in China were predominantly rural (>90\%) (14). Inclusion criteria of twins in the baseline survey were as follows: a) age, 6-60 y; b) both twins were available for the survey; and c) both twins (or parents/ guardians of children) agreed and consented to participate in the survey. In addition, they had no history of stroke, cardiovascular, renal, hepatic, or malignant diseases and were not pregnant or nursing. In the follow-up survey, eligible twins were those who met the following criteria: a) both twins participated in the baseline survey and b) both twins agreed and consented to participate in the follow-up study. Eligible twins were invited to a central office to complete a questionnaire interview, anthropometric measurements, and a blood drawn to measure fasting lipids.

Abbreviations: TC, total cholesterol; TG, triglyceride 
This report included 596 twin pairs who were aged 6-12 y at the baseline survey and who had completed follow-up study and zygosity determination. The twins had a mean follow-up interval of $6.7( \pm 0.6)$ y (range, 5.7-8.5 y). The study protocol was approved by the Institutional Review Boards at Children's Memorial Hospital and the Institute of Biomedicine, Anhui Medical University in Hefei, China.

Anthropometry and puberty. Body weight, height, and waist and hip circumferences were measured at both baseline and follow-up as previously described (14). During the follow-up visit, Tanner Stages (I-V) were assessed using established methods of visual inspection of pubic hair and genitals for boys and pubic hair and breasts for girls (15) by trained physicians.

Laboratory assays. Ten milliliters of blood sample was obtained from the cubital vein with the participant in the upright position during the morning (0700-0800 h) after an overnight fast. Serum was separated from blood cells in the field within $30 \mathrm{~min}$ of collection and kept frozen at $-20^{\circ} \mathrm{C}$. Baseline serum total cholesterol (TC), triglyceride (TG), and HDL cholesterol (HDLC) were measured on the XD-811 Semi-automatic Analyzer (Shanghai Antai Company, China). Follow-up serum TG, TC, and HDLC were measured on the Hitachi 7020 Automatic Analyzer. LDL cholesterol (LDLC) was estimated from TC, HDLC, and TG using the Friedewald formula (16). Twin zygosity [dizygotic (DZ) or monozygotic (MZ)] was determined using 10 autosomal polymorphic microsatellite markers as previously described (14).

Statistical analysis. The TC, TG, LDLC, and HDLC levels were normalized by natural logarithm transformation for all statistical testing as the original values were skewed. Mean values of the anthropometric measures and the lipids at the baseline and the follow-up are presented.

To evaluate the longitudinal changes of lipids between baseline and follow-up, we compared the distribution of the lipids and \% subjects with the highest tertiles of lipids at follow-up by the tertiles of each lipid (defined by age- and gender-specific values) at the baseline. ORs of the highest tertiles of lipids at follow-up and the corresponding 95\% CIs were obtained using the generalized estimating equations (GEE) to adjust within pair correlations, with adjustment for the follow-up age and gender. We also tested the linear trend across tertiles of serum lipid levels at the baseline. All analyses were performed using SAS software, version 9.0 (SAS Institute, Cary, NC).

To estimate the genetic and environmental contribution to each lipid (baseline and follow-up) and longitudinal lipid tracking, we fitted the bivariate Cholesky decompositions model using maximum likelihood estimation with structural equation modeling procedures $(\mathrm{Mx})$. Age-adjusted residuals of the lipids were used in all the modeling analyses. To address potential gender difference, we compared saturated model and no sex effect model first. If no significant sex difference was observed, the boys and girls could be analyzed together in the following analyses. In the full ACE model, additive genetic (A), common environmental (C), and unique environmental (E) components were structured by $\mathrm{Mx}$ (17). Then, we performed model comparisons by restricting certain components $(\mathrm{A}, \mathrm{C})$ as zero (i.e. $\mathrm{CE}$ model $=\mathrm{C}$ and $\mathrm{E}$, no genetic component; $\mathrm{AE}$ modal $=\mathrm{A}$ and $\mathrm{E}$, no common environmental component) and certain path coefficients: 1) the emergence of novel genetic effects at follow-up; 2 ) the emergence of novel common environmental effects at follow-up; 3 ) genetic correlation $\left(r_{\mathrm{G}}\right)$ between baseline and follow-up; 4) the common environmental correlation $\left(r_{\mathrm{C}}\right)$ between baseline and follow-up; or 5) the unique environmental correlation $\left(r_{\mathrm{E}}\right)$ between baseline and follow-up as zero. $\chi^{2}$ goodness of fit and Akaike's Information Criterion (AIC) were used for model comparisons. For the best-fitted model (i.e. model with nonsignificant $p$ value of $\chi^{2}$ test and the smallest AIC), we calculated the phenotypic correlations of lipids between baseline and follow-up as: $\left[r_{\mathrm{TP}}=r_{\mathrm{G} \times} \sqrt{h_{1}^{2} \times h_{2}^{2}}+r_{\mathrm{C} \times} \sqrt{c_{1}^{2} \times c_{2}^{2}}+r_{\mathrm{E} \times} \sqrt{e_{1}^{2} \times e_{2}^{2}}\right.$, where $r_{\mathrm{G}}, r_{\mathrm{C}}$, and $r_{\mathrm{E}}$ are the correlation estimates between baseline and follow-up traits for additive genetic component, common environmental, and unique environmental components. The $h_{1}{ }^{2}$ and $h_{2}{ }^{2}$ are additive genetic heritability estimates, $c_{1}{ }^{2}$ and $c_{2}{ }^{2}$ are common environmental component estimates, and $e_{1}{ }^{2}$ and $e_{2}{ }^{2}$ are the environmental component estimates for baseline and follow-up traits. The genetic, common, and unique environmental contributions to the phenotypic tracking correlations are $r_{\mathrm{G} \times} \sqrt{h_{1}{ }^{2} \times h_{2}{ }^{2}}$, $r_{\mathrm{C} \times} \sqrt{c_{1}^{2} \times c_{2}^{2}}$, and $r_{\mathrm{E} \times} \sqrt{e_{1}^{2} \times e_{2}^{2}}$, respectively.

\section{RESULTS}

This study included 596 twin pairs, including 331 pairs of MZ twins (male, 179; female, 152), 189 pairs of same-sex DZ twins (male, 117; female, 72), and 76 pairs of opposite-sex DZ twins. The distribution of the anthropometric characteristics and serum lipid levels measured at baseline and follow-up are presented in Table 1. As expected from growth and develop-
Table 1. General characteristics and serum lipid levels at baseline and follow-up surveys $(\mathrm{N}=596$ twin pairs $)$

\begin{tabular}{lrrr}
\hline & \multicolumn{2}{c}{ Mean \pm SD } & \\
\cline { 2 - 3 } \multicolumn{1}{c}{ Variables* } & \multicolumn{1}{c}{ Baseline } & \multicolumn{1}{c}{ Follow-up } & $p^{\dagger}$ \\
\hline Age $(\mathrm{y})$ & $9.2 \pm 1.4$ & $15.9 \pm 1.5$ & $<0.0001$ \\
Height $(\mathrm{cm})$ & $122.8 \pm 8.4$ & $156.3 \pm 8.0$ & $<0.0001$ \\
Weight $(\mathrm{kg})$ & $22.5 \pm 4.1$ & $46.4 \pm 7.5$ & $<0.0001$ \\
BMI $\left(\mathrm{kg} / \mathrm{m}^{2}\right)$ & $14.8 \pm 1.4$ & $18.9 \pm 2.3$ & $<0.0001$ \\
TC $(\mathrm{mM})$ & $3.30 \pm 0.68$ & $3.62 \pm 0.62$ & \\
Ln TC & $1.18 \pm 0.20$ & $1.27 \pm 0.17$ & $<0.0001$ \\
TG $(\mathrm{mM})$ & $0.65 \pm 0.34$ & $0.78 \pm 0.38$ & \\
Ln TG & $-0.54 \pm 0.45$ & $-0.36 \pm 0.44$ & $<0.0001$ \\
LDLC $(\mathrm{mM})$ & $1.56 \pm 0.59$ & $1.76 \pm 0.56$ & \\
Ln LDLC & $0.37 \pm 0.41$ & $0.51 \pm 0.33$ & $<0.0001$ \\
HDLC $(\mathrm{mM})$ & $1.44 \pm 0.38$ & $1.50 \pm 0.41$ & \\
Ln HDLC & $0.33 \pm 0.24$ & $0.37 \pm 0.27$ & 0.0003 \\
\hline
\end{tabular}

* For all statistical analyses in this article, the TC, TG, LDLC, and HDLC were transformed by natural logarithm, because of skewness.

$\dagger$ GEE were applied to compare the differences between baseline and follow-up survey for all variables, with adjustment of gender.

ment that occurs from childhood through adolescence, height, weight, and BMI at follow-up were all significantly higher than those at baseline $(p<0.0001)$, as were the serum lipids levels $(p<0.005)$.

The tracking of serum lipids from baseline to follow-up is displayed in Figure 1. Specifically, subjects were grouped into age- and gender- specific lipid tertiles (low, medium, and high) at the baseline survey. Next, each group's lipid levels at follow-up were plotted against age and Tanner stage at the follow-up, respectively. The participants with the highest tertile of lipid levels at the baseline tended to be high at the follow-up across all ages and Tanner stages, whereas subjects with the lowest tertile of lipid levels at the baseline tended to be low at the follow-up.

The ORs for being in the highest tertile of lipid level group at the follow-up in relation to age- and gender-specific tertiles of the lipid levels at the baseline are presented in Table 2 . Compared with subjects at the lowest tertiles at the baseline, those in the second and third tertiles were more likely to be in the highest tertile at the follow-up. For example, the ORs of the TC staying in the highest tertile at follow-up were 2.2 (95\% CI, 1.5-3.1) and 8.0 (95\% CI, 5.5-11.6) for twins who were in the second and third tertiles at the baseline, when compared with those who were in the lowest tertile of TC at the baseline ( $p$ trend $<0.001$ ). Similar statistically significant trends were also observed for TG, LDLC, and HDLC tracking. In addition, we found that higher tertiles of baseline $\mathrm{TC}$ were associated with the highest tertile of LDL and HDL at follow-up and that both LDLC and HDLC at the baseline were associated with TC at the follow-up.

No significant sex differences were founded for all lipids variables through sex-limited modeling (Table 3); therefore, we estimated the variance components by simply adjusting gender in all the structural equation models. The goodness of fit statistics from bivariate analyses of the lipids across baseline and follow-up visits is displayed in Table 4. The bestfitted models for TC, TG, LDLC, and HDLC were AE, ACE without novel common environmental effects at follow-up, 

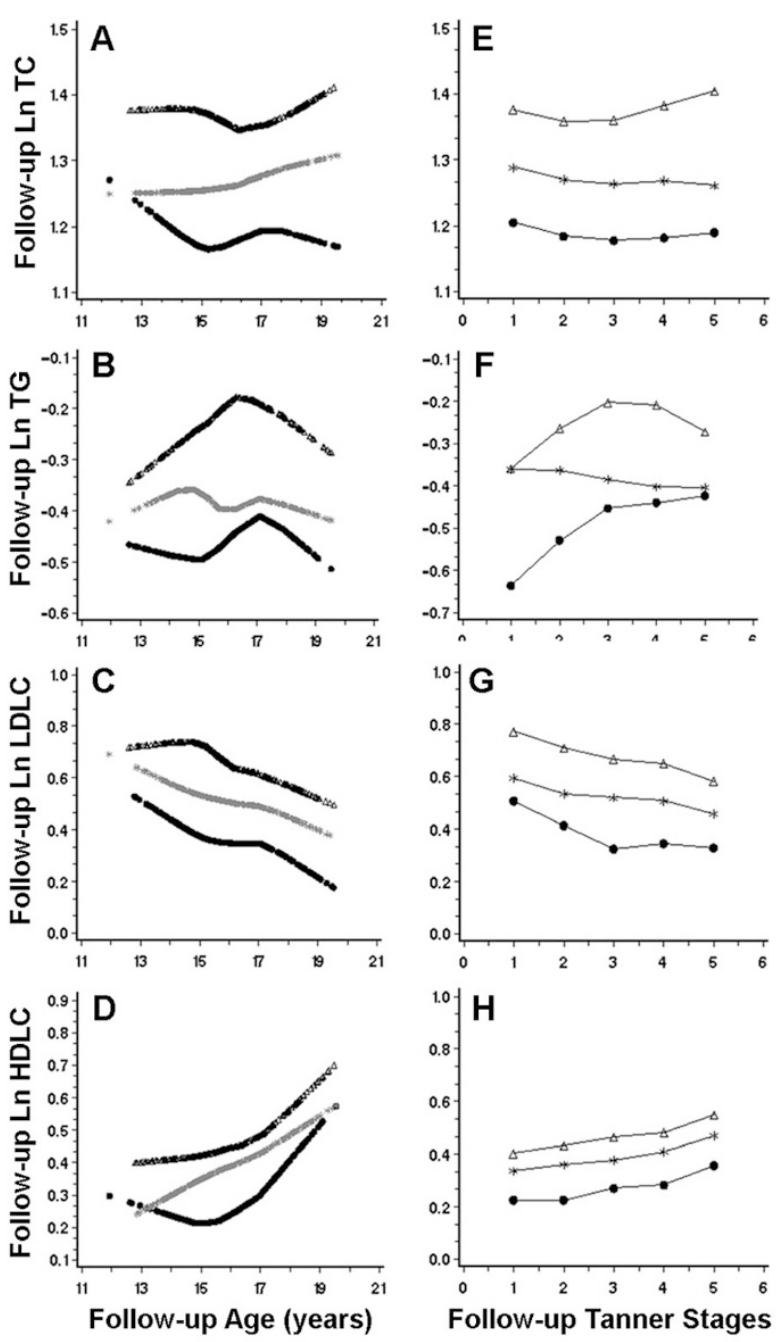

Figure 1. $(A-H)$ Smooth plots of lipid levels against follow-up ages and tanner stages stratified by age-and gender-specific lipid tertiles at baseline (TC, TG, LDLC, and HDLC were normalized by natural logarithm transformation for all statistical tests because of skewness). $\boldsymbol{\Delta}$, high baseline tertile; $*$, medium baseline tertile; $\bullet$, low baseline tertile.

ACE without common environmental correlation (i.e. no $r_{\mathrm{G}}$ ), and full ACE, respectively, which were the basis for the parameter estimates of Figure 2.

As shown in Table 4, the hypothesis of no novel genetic effects that emerged at follow-up visit was statistically rejected for all lipids except TG $(p=0.074)$, which indicated the emergences of some new genetic influences for these lipids at follow-up visit. On the contrary, no novel common environmental effects emerged at follow-up for all lipids.

The estimates for the heritability $\left(h^{2}\right)$ of various serum lipids at the baseline and follow-up, variance component correlation coefficients, and the genetic and environmental contributions to the total phenotypic correlations are presented in Figures $2 A-D$. Heritability estimates for TC and LDLC were relatively stable from the baseline to follow-up (TC, 0.77-0.83; LDLC, 0.59-0.60). Larger increases over time were observed in the heritability of TG $(0.40-0.69)$, and there was a corresponding decrease in the effect of common environmental factors over time for this trait $(0.26-0.07)$. In contrast, the heritability estimates for HDLC decreased moderately over time from childhood into adolescence (0.650.39 ), with a corresponding increase in common environmental components. The results were consistent with the analyses of within-pair intra-class correlations (Table 5). For unique environmental components, we observed mild to moderate decreases from the baseline to follow-up visit (TC, 0.23-0.17; TG, 0.34-0.23; LDLC, 0.33-0.11; and HDLC, 0.18-0.13).

These figures further demonstrated that although genetic influences for all the lipids were significantly correlated over time from childhood through adolescence, no significant correlations were found for common and unique environmental components. The phenotypic correlations of lipids between the baseline and follow-up visits ranged from 0.26 to 0.53 , with the highest value for TC and the lowest value for TG. Major contributions to these phenotypic correlations were genetic influences. For example, genetic factors accounted for $98 \%$ of the tracking correlation for TC $(=0.52 / 0.53)$. However, for HDLC, common environmental influences also contributed to the phenotypic correlations $(29 \%=0.10 / 0.35)$. The unique environment contribution to phenotypic correlation was very small for all the lipids analyzed.

\section{DISCUSSION}

Serum lipid levels are important predictors of coronary artery disease risk in adults $(1,2)$, and the risk of disease can be decreased by lowering total and LDLC or by increasing HDLC (18-20). Tracking of serum lipid levels from childhood to adulthood has been established (21). However, the degree of tracking from childhood to adolescence and the underlying genetic and environmental contributions on the phenotypic tracking are not well understood. Our study has contributed the following new findings.

First, the lipid levels at the follow-up are significantly higher than those at the baseline, which are inconsistent with previous studies (22). The reason for the increased lipids levels after an $\sim 6-\mathrm{y}$ interval may be attributed to the rapid economic, nutritional, and lifestyle changes observed in China over the recent decades $(23,24)$. In particular, diet changes that include more animal-derived products and less traditional food consumption (rice and vegetables) likely a direct result of the rapid economic growth $(25,26)$ and might explain the observed changes in lipid levels. A previous study based on a rural Chinese population demonstrated that hyperlipidemia was positively associated with total energy, total fat, and saturated fatty acid intakes and negatively associated with physical activity and total fiber intake (27). Furthermore, puberty, an important developmental period accompanied by rapid growth in body size and sexual maturation may influence the lipids levels and provide another explanation for the lipid changes between baseline and follow-up surveys. Bertrais et al. (28) also reported that TC and TG were associated with sexual maturity independently of age in adolescence.

Second, we observed all the lipids variables (TG, TC, LDLC, and HDLC) appear to be under considerable additive genetic and unique environmental influence. However, phe- 
Table 2. Longitudinal relationship of the highest tertile of lipids at follow-up with each tertile of lipids at baseline

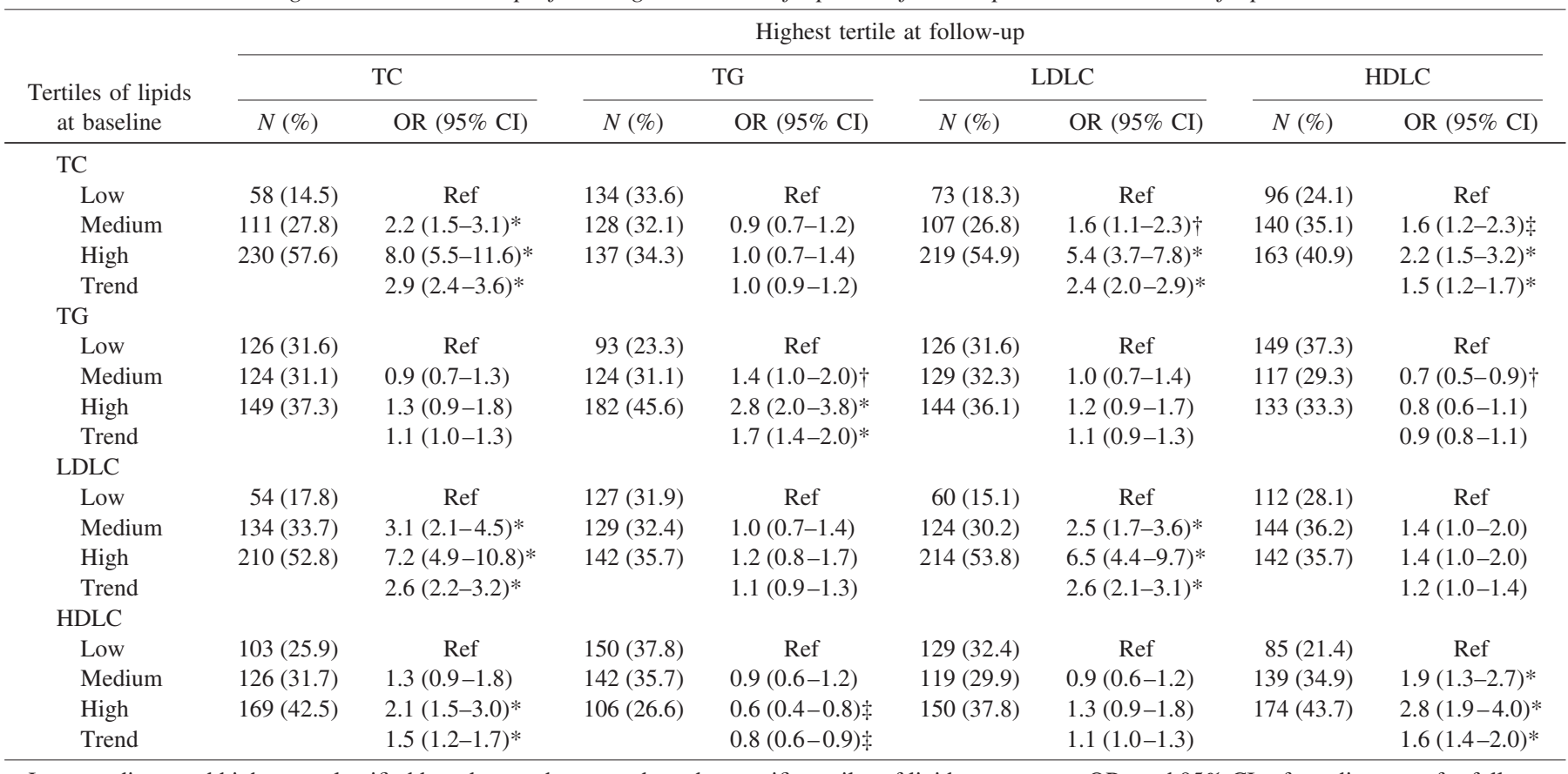

Low, medium, and high were classified based on each age- and gender-specific tertiles of lipids parameters. ORs and 95\% CIs after adjustment for follow-up age and gender.

$* p<0.001$.

$\dagger p<0.05$.

$\ddagger p<0.01$

Table 3. Goodness of fit statistics from sex-limited models of the lipids variables

\begin{tabular}{|c|c|c|c|c|c|}
\hline Lipids variables & Model & AIC & $\Delta \mathrm{df}$ & $\Delta \mathrm{AIC}$ & $p$ \\
\hline \multirow[t]{2}{*}{ Baseline TC } & Saturated & -3111.2 & 1 & -1.8 & 0.660 \\
\hline & No sex effect & -3113.0 & & & \\
\hline \multirow[t]{2}{*}{ Baseline TG } & Saturated & -1123.7 & 1 & -1.9 & 0.794 \\
\hline & No sex effect & -1125.6 & & & \\
\hline \multirow[t]{2}{*}{ Baseline LDL } & Saturated & -1373.9 & 1 & -1.8 & 0.639 \\
\hline & No sex effect & -1365.7 & & & \\
\hline \multirow[t]{2}{*}{ Baseline HDL } & Saturated & -2735.9 & 1 & -1.9 & 0.732 \\
\hline & No sex effect & -2737.8 & & & \\
\hline \multirow[t]{2}{*}{ Follow-up TC } & Saturated & -3688.9 & 1 & -1.2 & 0.363 \\
\hline & No sex effect & -3690.0 & & & \\
\hline \multirow[t]{2}{*}{ Follow-up TG } & Saturated & -1282.6 & 1 & -1.9 & 0.769 \\
\hline & No sex effect & -1284.5 & & & \\
\hline \multirow[t]{2}{*}{ Follow-up LDL } & Saturated & -2295.3 & 1 & -1.2 & 0.364 \\
\hline & No sex effect & -2296.5 & & & \\
\hline \multirow[t]{2}{*}{ Follow-up HDL } & Saturated & -2842.0 & 1 & -1.7 & 0.571 \\
\hline & No sex effect & -2843.7 & & & \\
\hline
\end{tabular}

$\Delta$, difference; df, degrees of freedom.

notypic variance of HDLC and LDLC at the follow-up seems to be partly explained by common environmental factors besides genetic and unique environmental influences. The findings are in agreement with previous study, which suggests additive genetic and unique environmental influences contributed to the majority of the phenotypic variance of TG, LDLC, and HDLC (29). Interestingly, the additive genetic factors of TG were increased over time and the common environmental factors were decreased. However, the opposite trends were observed in HDLC variance estimates. Our previous study based on adult female twins reported that the heritability estimates of TG and HDLC were 0.50 and 0.22 , respectively
(30). These estimates were similar to the heritability estimates at the follow-up survey. Of note, most of the subjects were adolescents (age $>12$ y) at the follow-up survey, whereas most of the subjects at the baseline survey were preadolescents (age $\leq 12$ y). Our data suggest that puberty specific genetic factors may partly explain the change of variance estimates from baseline to follow-up surveys. It is also likely that the shared familial environmental factors (such as diet type, meal size, physical activity and so on) of twin pairs changed from baseline to follow-up surveys may thus provide another explanation for the observed results.

Third, we observed differences in variance component changes from the baseline to the follow-up visits between HDLC and the other lipids. The HDLC is known as a type of good lipid, whereas other lipids, i.e. TC, LDLC, and TG, are regarded as bad types of lipids. Our data suggest that HDLC may be under different genetic and environmental influences from the other lipids. Previous studies suggested that the HDLC was the only lipid variable that could be influenced by shared environmental factors both in adults and adolescents $(10,31)$. We speculate that shared environmental influences within a family, such as socioeconomic status or diet type, may influence HDLC more than the other lipid variables during adolescence.

Fourth, we observed longitudinal tracking for all of the lipids as analyzed by both tracking (persistence at corresponding levels; Fig. 1) and \% shift in tertiles from the baseline to the follow-up (Table 2). The tracking for TC and LDLC was greater than for HDLC and TG. These findings were comparable with those reported in Caucasians $(5,29)$. For instance, 
Table 4. Goodness of fit statistics from bivariate analyses of the lipids variables between baseline and follow-up visits

\begin{tabular}{|c|c|c|c|c|c|}
\hline Lipids & Model & AIC & $\Delta \mathrm{df}$ & $\Delta \mathrm{AIC}$ & $p$ \\
\hline \multirow[t]{8}{*}{ TC } & ACE & -7125.6 & & & \\
\hline & $\mathrm{AE}^{*}$ & -7128.1 & 3 & -2.5 & 0.316 \\
\hline & $\mathrm{CE}$ & -6973.5 & 3 & 152.1 & $<0.001$ \\
\hline & ACE without novel genetic effects at follow-up & -7115.9 & 1 & 9.8 & 0.001 \\
\hline & ACE without novel common environmental effects at follow-up* & -7127.3 & 1 & -1.7 & 0.562 \\
\hline & No $r_{\mathrm{G}} \mathrm{ACE}$ & -7049.7 & 1 & 76.0 & $<0.001$ \\
\hline & No $r_{\mathrm{C}} \mathrm{ACE}$ & -7127.6 & 1 & -1.9 & 0.842 \\
\hline & No $r_{\mathrm{E}} \mathrm{ACE}$ & -7127.6 & 1 & -1.9 & 0.806 \\
\hline \multirow[t]{8}{*}{ TG } & $\mathrm{ACE}$ & -2524.6 & & & \\
\hline & $\mathrm{AE}$ & -2513.3 & 3 & 11.3 & 0.001 \\
\hline & $\mathrm{CE}$ & -2435.7 & 3 & 88.9 & $<0.001$ \\
\hline & ACE without novel genetic effects at follow-up & -2523.3 & 1 & 1.3 & 0.071 \\
\hline & ACE without novel common environmental effects at follow-up & -2526.6 & 1 & -2 & 1.000 \\
\hline & No $r_{\mathrm{G}}$ ACE & -2493.4 & 1 & 31.2 & $<0.001$ \\
\hline & No $r_{\mathrm{C}} \mathrm{ACE}$ & -2520.7 & 1 & 3.9 & 0.015 \\
\hline & No $r_{\mathrm{E}} \mathrm{ACE}$ & -2526.3 & 1 & -1.7 & 0.591 \\
\hline \multirow[t]{8}{*}{ LDLC } & $\mathrm{ACE}$ & -3863.4 & & & \\
\hline & $\mathrm{AE}$ & -3852.0 & 3 & 11.3 & 0.001 \\
\hline & $\mathrm{CE}$ & -3704.2 & 3 & 159.2 & $<0.001$ \\
\hline & ACE without novel genetic effects at follow-up & -3857.8 & 1 & 5.6 & 0.006 \\
\hline & ACE without novel common environmental effects at follow-up & -3864.3 & 1 & -0.9 & 0.289 \\
\hline & No $r_{\mathrm{G}} \mathrm{ACE}$ & -3814.4 & 1 & 49.0 & $<0.001$ \\
\hline & No $r_{\mathrm{C}} \mathrm{ACE}^{*}$ & -3865.3 & 1 & -1.9 & 0.712 \\
\hline & No $r_{\mathrm{E}} \mathrm{ACE}$ & -3862.9 & 1 & 0.5 & 0.113 \\
\hline \multirow[t]{8}{*}{ HDLC } & $\mathrm{ACE}^{*}$ & -5742.0 & & & \\
\hline & $\mathrm{AE}$ & -5706.2 & 3 & 35.8 & $<0.001$ \\
\hline & $\mathrm{CE}$ & -5608.7 & 3 & 133.3 & $<0.001$ \\
\hline & ACE without novel genetic effects at follow-up & -5707.2 & 1 & 34.8 & $<0.001$ \\
\hline & ACE without novel common environmental effects at follow-up & -5740.8 & 1 & 1.2 & 0.073 \\
\hline & No $r_{\mathrm{G}}$ ACE & -5722.9 & 1 & 19.1 & $<0.001$ \\
\hline & No $r_{\mathrm{C}}$ ACE & -5741.1 & 1 & 0.954 & 0.086 \\
\hline & No $r_{\mathrm{E}}$ ACE & -5740.7 & 1 & 1.4 & 0.066 \\
\hline
\end{tabular}

* The best-fitting model.

$\Delta$, difference; df, degrees of freedom.

Boulton et al. (5) reported tracking coefficients for TC of $0.28-0.49$ between 1 and 15 y of age, $0.30-0.64$ between $2-8$ and $15 \mathrm{y}$ of age, and $0.71-0.78$ between 13 and $15 \mathrm{y}$ of age in an Australian sample. The pattern was similar for LDLC but lower for HDLC. The tracking pattern for LDLC was similar to TC, whereas less tracking was observed for HDLC than for TC and LDLC. In addition, Porkka and Viikari (32) found that the tracking of TG was lower than other lipids in Finnish children and young adults.

Previous longitudinal twin studies based on Caucasian samples reported that more than one genetic factor influences HDLC, LDLC, TC, and TG over time at ages 12, 14, and $16 \mathrm{y}$, i.e. there are developmental changes in the genes or environmental factors affecting plasma lipids during adolescence $(10,13)$. Our data suggest emergence of novel genetic influence for all lipid variables (except for TG) from baseline (6-12 y) to follow-up surveys ( $>12 \mathrm{y})$. A potential explanation for the emergence of novel genetic effects is that sexual hormonal changes during puberty, which may affect gene expressions and that in turn influence individual differences in lipids.

Finally, our study demonstrated that individual lipid levels were under the control of both genetic and environmental factors, which is consistent with previous reports (10,33-35). We further demonstrated that the majority of phenotypic tracking of the lipids from childhood through adolescence was attributed to genetic influences. In contrast, common and unique environmental components contributed minimally to tracking for all the lipids except for HDLC. In view of the importance of genetic factors in the lipid tracking from childhood to adolescence, the children with higher lipids values during childhood and with parents of higher lipids values, particularly for TC and LDL may be at higher risk of dyslipidemia. Those children may benefit from more close monitoring and early intervention to prevent or mitigate dyslipidemia later in life.

Our study has several strengths. First, the twin design has a long-standing history of studying the relative roles of genetic and environmental factors in the development of disease and phenotypic traits. Second, the study subjects were recruited from two adjacent rural areas of China that are relatively homogeneous with regard to the dietary intakes and physical activity. Finally, the longitudinal twin study design, the age range at baseline (6-12 y) and follow-up (12-19 y), provides a unique opportunity to investigate the genetic and environmental contributions to lipid profile tracking from childhood through adolescence in a lean, rural Chinese population, which has not been examined earlier.

In summary, we have demonstrated significant tracking for all the lipids analyzed from childhood through adolescence in 

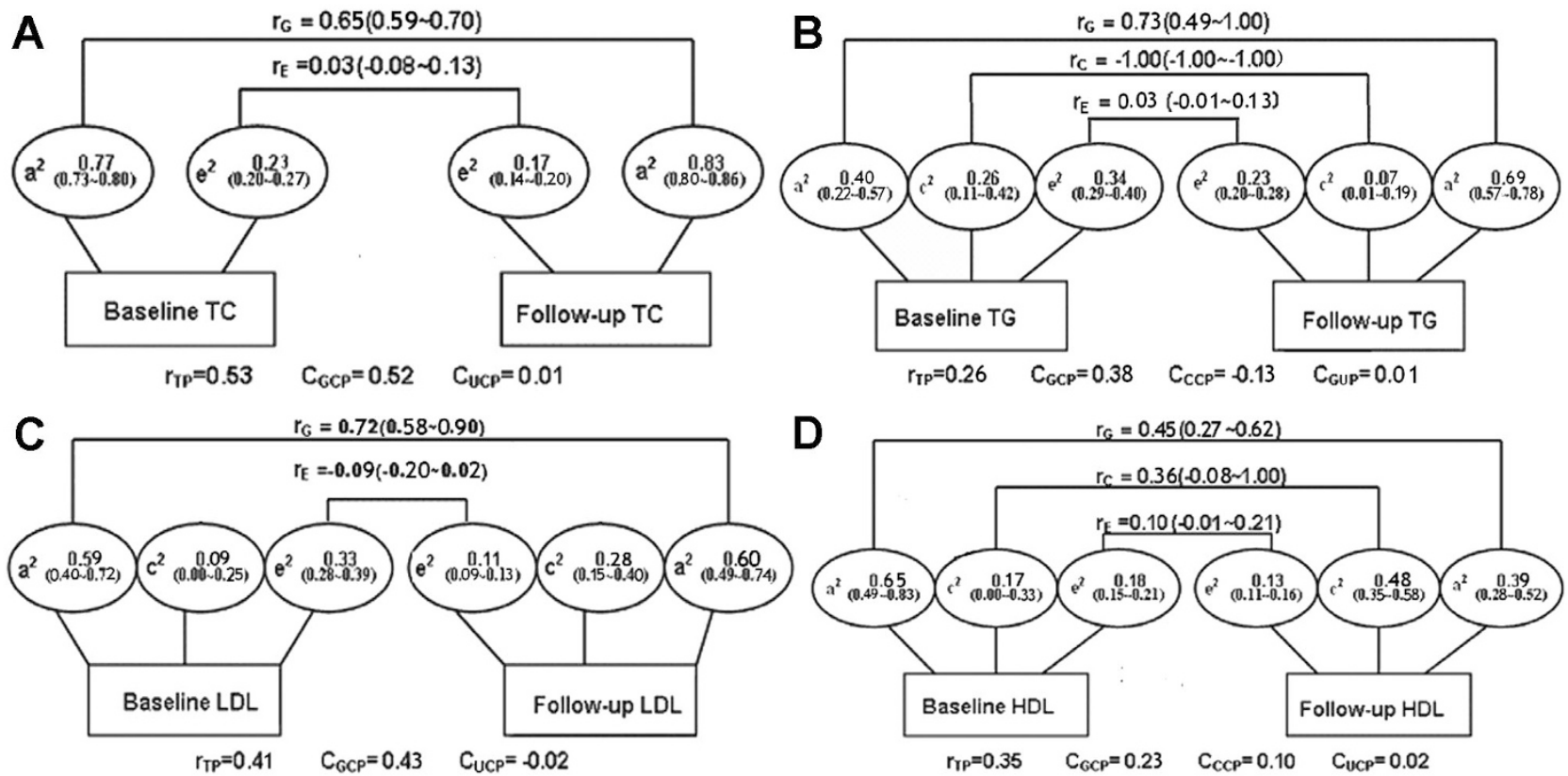

Figure 2. A, Genetic and environmental contributions to tracking of TC in 520 same-sex adolescent twin pairs adjusted for sex and age. AE model was the best-fitting model for the bivariate analyses between baseline and follow-up TC; $r_{\mathrm{G}}$, and $r_{\mathrm{E}}$ denote genetic and unique environmental correlations, respectively; $a^{2}$ and $e^{2}$ denote percentage of total phenotypic variance accounted for genetic factors and unique environmental factors; $r_{\mathrm{TP}}$, total phenotypic correlation; $C_{\mathrm{GCP}}$, genetic contribution to total phenotypic correlation; $C_{\mathrm{UCP}}$, unique environmental contribution to total phenotypic correlation. $B$, Genetic and environmental contributions to tracking of TG in 520 same-sex adolescent twin pairs adjusted for sex and age. No unique environmental correlation ACE model was the best-fitting model for the bivariate analyses between baseline and follow-up TG; $r_{\mathrm{G}}, r_{\mathrm{C}}$, and $r_{\mathrm{E}}$ denote genetic, common environmental, and unique environmental correlations, respectively; $a^{2}, c^{2}$, and $e^{2}$ denote percentage of total phenotypic variance accounted for genetic factors, common environmental, and unique environmental factors; $r_{\mathrm{TP}}$, total phenotypic correlation; $C_{\mathrm{GCP}}$, genetic contribution to total phenotypic correlation; $C_{\mathrm{CCP}}$, common environmental contribution to total phenotypic correlation; $C_{\mathrm{UCP}}$, unique environmental contribution to total phenotypic correlation. $C$, genetic and environmental contributions to tracking of LDLC in 520 same-sex adolescent twin pairs adjusted for sex and age. No common environmental correlation ACE model was the best-fitting model for the bivariate analyses between baseline and follow-up LDLC; $r_{\mathrm{G}}, r_{\mathrm{C}}$, and $r_{\mathrm{E}}$ denote genetic, common environmental, and unique environmental correlations, respectively; $a^{2}, c^{2}$, and $e^{2}$ denote percentage of total phenotypic variance accounted for genetic factors, common environmental, and unique environmental factors; $r_{\mathrm{TP}}$, total phenotypic correlation; $C_{\mathrm{GCP}}$, genetic contribution to total phenotypic correlation; $C_{\mathrm{CCP}}$, common environmental contribution to total phenotypic correlation; $C_{\mathrm{UCP}}$, unique environmental contribution to total phenotypic correlation. $D$, Genetic and environmental contributions to tracking of HDLC in 520 same-sex adolescent twin pairs adjusted for sex and age. Full ACE model was the best-fitting model for the bivariate analyses between baseline and follow-up HDLC; $r_{\mathrm{G}}, r_{\mathrm{C}}$, and $r_{\mathrm{E}}$ denote genetic, common environmental, and unique environmental correlations, respectively; $a^{2}, c^{2}$, and $e^{2}$ denote percentage of total phenotypic variance accounted for genetic factors, common environmental, and unique environmental factors; $r_{\mathrm{TP}}$, total phenotypic correlation; $C_{\mathrm{GCP}}$, genetic contribution to total phenotypic correlation; $C_{\mathrm{CCP}}$, common environmental contribution to total phenotypic correlation; $C_{\mathrm{UCP}}$, unique environmental contribution to total phenotypic correlation.

Table 5. Intraclass correlation coefficient for lipids variables in $M Z(\mathrm{n}=331$ pairs $)$ and $D Z(\mathrm{n}=265$ pairs $)$

\begin{tabular}{|c|c|c|c|c|}
\hline \multirow[b]{3}{*}{ Variables } & \multicolumn{4}{|c|}{ Interclass correlation } \\
\hline & \multicolumn{2}{|c|}{ MZ } & \multicolumn{2}{|c|}{$\mathrm{DZ}$} \\
\hline & $r$ & $p$ & $r$ & $p$ \\
\hline \multicolumn{5}{|l|}{ Baseline } \\
\hline $\mathrm{TC}$ & 0.83 & $<0.001$ & 0.45 & $<0.001$ \\
\hline TG & 0.66 & $<0.001$ & 0.44 & $<0.001$ \\
\hline LDLC & 0.67 & $<0.001$ & 0.40 & $<0.001$ \\
\hline HDLC & 0.82 & $<0.001$ & 0.51 & $<0.001$ \\
\hline \multicolumn{5}{|l|}{ Follow-up } \\
\hline $\mathrm{TC}$ & 0.77 & $<0.001$ & 0.46 & $<0.001$ \\
\hline TG & 0.76 & $<0.001$ & 0.39 & $<0.001$ \\
\hline LDLC & 0.89 & $<0.001$ & 0.59 & $<0.001$ \\
\hline HDLC & 0.86 & $<0.001$ & 0.67 & $<0.001$ \\
\hline
\end{tabular}

* Age- and sex-adjusted Pearson partial correlation analysis was used to continuous variables.

this population-based longitudinal Chinese twin cohort. Genetic factors were the predominant contributor to the phenotypic tracking for TC, TG, and LDLC. For HDLC, both genetic and common environmental factors contributed to the phenotypic tracking. Our study underscores the importance of considering both environmental and genetic factors in studying the etiology of dyslipidemia. Further investigation of specific genetic and environmental factors associated with lipid levels and tracking from childhood through adolescence will provide useful insight into the underlying biologic mechanisms in the development of dyslipidemia and its related diseases and help design preventive strategies.

Acknowledgments. We thank the assistance and cooperation of the faculty and staff of the Anhui Institute of Biomedicine, Anhui Medical University, and thank all study participants for their support.

\section{REFERENCES}

1. Ballantyne C, Arroll B, Shepherd J 2005 Lipids and CVD management: towards a global consensus. Eur Heart J 26:2224-2231

2. Khoo KL, Tan H, Liew YM, Deslypere JP, Janus E 2003 Lipids and coronary heart disease in Asia. Atherosclerosis 169:1-10 
3. Berenson GS, Srinivasan SR, Bao W, Newman WP III, Tracy RE, Wattigney WA 1998 Association between multiple cardiovascular risk factors and atherosclerosis in children and young adults. The Bogalusa Heart Study. N Engl J Med 338:1650-1656

4. Strong JP 1991 The natural history of atherosclerosis in childhood. Ann N Y Acad Sci 623:9-15

5. Boulton TJ, Magarey AM, Cockington RA 1995 Tracking of serum lipids and dietary energy, fat and calcium intake from 1 to 15 years. Acta Paediatr 84:10501055

6. Twisk JW, Kemper HC, Mellenbergh DJ, van Mechelen W 1996 Factors influencing tracking of cholesterol and high-density lipoprotein: the Amsterdam Growth and Health Study. Prev Med 25:355-364

7. Kirkwood L, Aldujaili E, Drummond S 2007 Effects of advice on dietary intake and/or physical activity on body composition, blood lipids and insulin resistance following a low-fat, sucrose-containing, high-carbohydrate, energy-restricted diet. Int J Food Sci Nutr 58:383-397

8. Mbalilaki JA, Hellènius ML, Masesa Z, Høstmark AT, Sundquist J, Strømme SB 2007 Physical activity and blood lipids in rural and urban Tanzanians. Nutr Metab Cardiovasc Dis 17:344-348

9. Li GP, Wang JY, Yan SK, Chen BS, Xue H, Wu G 2004 Genetic effect of two polymorphisms in the apolipoprotein A5 gene and apolipoprotein $\mathrm{C} 3$ gene on serum lipids and lipoproteins levels in a Chinese population. Clin Genet 65:470-476

10. Middelberg RP, Martin NG, Whitfield JB 2007 A longitudinal genetic study of plasma lipids in adolescent twins. Twin Res Hum Genet 10:127-135

11. Callas N, Poveda E, Baracaldo C, Hernández P, Castillo C, Guerra M 2007 [Genetic polymorphism of the $\mathrm{E}$ apolipoprotein in school age children: comparison with levels of plasma lipids and apolipoproteins.] Biomedica 27:526-536

12. Jensen MK, Pai JK, Mukamal KJ, Overvad K, Rimm EB 2007 Common genetic variation in the ATP-binding cassette transporter A1, plasma lipids, and risk of coronary heart disease. Atherosclerosis 195:e172-e180

13. Nance WE, Bodurtha J, Eaves LJ, Hewitt J, Maes H, Segrest J, Meyer J, Neale M, Schieken R 1998 Models for the longitudinal genetic analysis of same-age twins: application to HDL cholesterol. Twin Res 1:3-8

14. Wang B, Necheles J, Ouyang F, Ma W, Li Z, Liu X, Yang J, Xing H, Xu X, Wang X 2007 Monozygotic co-twin analyses of body composition measurements and serum lipids. Prev Med 45:358-365

15. Marshall WA, Tanner JM 1970 Variations in the pattern of pubertal changes in boys. Arch Dis Child 45:13-23

16. Friedewald WT, Fredrickson DS 1972 Estimation of the concentration of lowdensity lipoprotein cholesterol in plasma, without use of the preparative ultracentrifuge. Clin Chem 18:499-502

17. Neale MC, Cardon LR 1992 Methodology for Genetic Studies of Twins and Families. Kluwer Academic Publishers, Dordrecht, The Netherlands

18. Superko HR, King S III 2008 Lipid management to reduce cardiovascular risk: a new strategy is required. Circulation 117:560-568

19. Lopez VA, Franklin SS, Tang S, Wong ND 2007 Coronary heart disease events preventable by control of blood pressure and lipids in US adults with hypertension. J Clin Hypertens (Greenwich) 9:436-443
20. Larosa JH, Larosa JC 2006 Coronary heart disease prevention in women: focus on lipids. Minerva Cardioangiol 54:311-322

21. Adams C, Burke V, Beilin LJ 2005 Cholesterol tracking from childhood to adult mid-life in children from the Busselton study. Acta Paediatr 94:275-280

22. Brotons C, Ribera A, Perich RM, Abrodos D, Magana P, Pablo S, Terradas D, Fernandez F, Permanyer G 1998 Worldwide distribution of blood lipids and lipoproteins in childhood and adolescence: a review study. Atherosclerosis 139:1-9

23. Du S, Lu B, Zhai F, Popkin BM 2002 A new stage of the nutrition transition in China. Public Health Nutr 5:169-174

24. Wang CN, Liang Z, Wei P, Liu P, Yu JX, Zhang DM, Ma FL 2002 Changes in dietary patterns and certain nutrition-related diseases in urban and rural residents of Jiangsu Province, China, during the 1990s. Biomed Environ Sci 15:271-276

25. Zhai F, Wang H, Du S, He Y, Wang Z, Ge K, Popkin BM 2007 Lifespan nutrition and changing socio-economic conditions in China. Asia Pac J Clin Nutr 16:374-382

26. Wang Z, Zhai F, He Y, Wang H 2008 [Influence of family income on dietary nutrients intake and dietary structure in China.] Wei Sheng Yan Jiu 37:62-64

27. Ruixing Y, Jinzhen W, Yaoheng H, Jing T, Hai W, Muyan L, Yiyang L, Dongmei F, Hanjun Y, Yuming C 2008 Associations of diet and lifestyle with hyperlipidemia for middle-aged and elderly persons among the Guangxi Bai Ku Yao and Han populations. J Am Diet Assoc 108:970-976

28. Bertrais S, Balkau B, Charles MA, Vol S, Calvet C, Tichet J, Eschwege E 2000 Puberty-associated differences in total cholesterol and triglyceride levels according to sex in French children aged 10-13 years. Ann Epidemiol 10:316-323

29. Benyamin B, Sorensen TI, Schousboe K, Fenger M, Visscher PM, Kyvik KO 2007 Are there common genetic and environmental factors behind the endophenotypes associated with the metabolic syndrome? Diabetologia 50:1880-1888

30. Zhang S, Liu X, Yu Y, Hong X, Christoffel K, Wang B, Tsai HJ, Li Z, Liu X, Tang G, Xing H, Brickman W, Zimmerman D, Xu X, Wang X 2009 Genetic and environmental contributions to phenotypic components of metabolic syndrome: a population-based twin study. Obesity (Silver Spring) 17:1581-1587

31. Goode EL, Cherny SS, Christian JC, Jarvik GP, de Andrade M 2007 Heritability of longitudinal measures of body mass index and lipid and lipoprotein levels in aging twins. Twin Res Hum Genet 10:703-711

32. Porkka KV, Viikari JS 1995 Tracking of serum lipids in children; association with the absolute lipid level-the cardiovascular risk in young Finns study. J Clin Epidemiol 48:221-228

33. Poulsen P, Vaag A, Kyvik K, Beck-Nielsen H 2001 Genetic versus environmental aetiology of the metabolic syndrome among male and female twins. Diabetologia 44:537-543

34. Heller DA, de Faire U, Pedersen NL, Dahlén G, McClearn GE 1993 Genetic and environmental influences on serum lipid levels in twins. N Engl J Med 328:11501156

35. Bayoumi RA, Al-Yahyaee SA, Albarwani SA, Rizvi SG, Al-Hadabi S, Al-Ubaidi FF, Al-Hinai AT, Al-Kindi MN, Adnan HT, Al-Barwany HS, Comuzzie AG, Cai G, Lopez-Alvarenga JC, Hassan MO 2007 Heritability of determinants of the metabolic syndrome among healthy Arabs of the Oman family study. Obesity (Silver Spring) $15: 551-556$ 